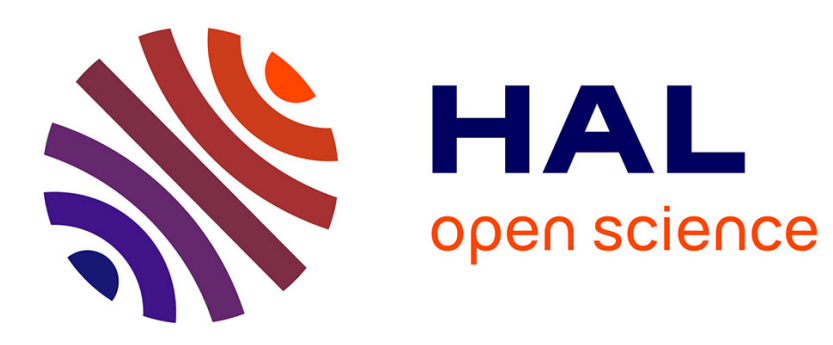

\title{
The use of silicon photomultipliers for very high energy gamma ray astronomy: the optical issues
}

Alexandre Cadu, Arnaud Le Padellec, Khalil Jradi, Denis Pellion, Robert

Bazer-Bachi

\section{- To cite this version:}

Alexandre Cadu, Arnaud Le Padellec, Khalil Jradi, Denis Pellion, Robert Bazer-Bachi. The use of silicon photomultipliers for very high energy gamma ray astronomy: the optical issues. Experimental Astronomy, 2012, 10.1007/s10686-012-9317-y . hal-00783740

\author{
HAL Id: hal-00783740 \\ https://hal.science/hal-00783740
}

Submitted on 4 Feb 2013

HAL is a multi-disciplinary open access archive for the deposit and dissemination of scientific research documents, whether they are published or not. The documents may come from teaching and research institutions in France or abroad, or from public or private research centers.
L'archive ouverte pluridisciplinaire HAL, est destinée au dépôt et à la diffusion de documents scientifiques de niveau recherche, publiés ou non, émanant des établissements d'enseignement et de recherche français ou étrangers, des laboratoires publics ou privés. 


\title{
The use of silicon photomultipliers for very high energy gamma ray astronomy: the optical issues
}

\author{
A. Cadu • A. Le Padellec • K. Jradi • \\ D. Pellion - A. R. Bazer-Bachi
}

Received: 15 October 2011 / Accepted: 10 September 2012

(C) Springer Science+Business Media B.V. 2012

\begin{abstract}
Due to its sensitivity and speed, the detector still widely used in Cerenkov astrophysics experiments remains the Photo-Multiplier Tube (PMT). However, there are some disadvantages to the PMT technology: the rather poor quantum efficiency, the use of high voltages, the high cost when used in large number in a matrix arrangement and a subsequent large weight. Hence, we have investigated the possibility to design future Cerenkov telescope cameras based on solid state technology, specifically Geiger Avalanche PhotoDiodes (G-APD's). This paper describes our extensive simulations to design the optical setup to be employed with G-APD's. We also discuss the reflector configurations, pixel layouts, light concentrators, microlens arrays and spectral efficiencies to optimize light collection. The electronic aspects of our work were presented in a recent companion paper (Pellion et al., Exp. Astron. 27(3):187, 2010).
\end{abstract}

Keywords G-APD • Optics • Microlenses • Cerenkov effect • VHE astronomy

\section{Introduction}

Gamma rays are produced in a large variety of objects and environments in the Universe, such as pulsars, plerions and active galactic nuclei. Typical photon

\footnotetext{
A. Cadu $(\bowtie) \cdot$ A. Le Padellec · K. Jradi · D. Pellion · A. R. Bazer-Bachi Université de Toulouse, UPS., Institut de Recherche en Astrophysique et Planétologie, 9 , avenue du Colonel Roche, 31028 Toulouse Cedex 9, France e-mail: alexandre.cadu@irap.omp.eu
}

A. Cadu - A. Le Padellec · K. Jradi - D. Pellion · A. R. Bazer-Bachi CNRS, UMR 5277, 31028 Toulouse, France 
fluxes in the very high-energy (VHE) gamma-ray domain are below one photon per square kilometer per second, and consequently, the effective detection area needs to be very large in order to study related sources with sufficient statistics. Such surfaces can be achieved by using the Earth's atmosphere as a detection device, where incoming VHE gamma-rays create electromagnetic air showers that are subsequently observable through their induced Cerenkov light. To put numbers on the Cerenkov photon flux that originates from a $100 \mathrm{GeV}$ photon, one would expect about 5 visible (or near UV) photons per square meter for the duration of a Cerenkov flash which lasts for about 2 nanoseconds and spreads over an ellipse of hundreds of meters for the minor axis, at sea level. This requires fast but also very sensitive detectors, and at the present time, the conventional detection scheme is based on PMT's. These latter are very well documented in the literature $[1,2]$.

In recent years, however, an alternative technology based on solid state detectors has emerged [3,4] which has the potential to overcome some of the drawbacks of PMTs, such as their relatively large weight and size, the need for high voltage supplies and their limited quantum efficiency in terms of amplitude and of spectral width. These solid state detectors are based on single APD's and are realized by silicon doped $\mathrm{P} / \mathrm{N}$ junctions. Their operating principle is rather simple $[4,5]$. A photon arrives in the depletion region -or space charge region- causes the ejection of an electron from the valence band to the conduction band with the subsequent appearance of an electron / hole pair in the crystal lattice. Since the diode is polarized, the carriers move under the effect of an electric field to generate secondary pairs which create a measurable current across the component. Moreover, APD's operating beyond the breakdown voltage (typically several tens of volts) offer the advantage of a large gain, up to $10^{6}$ electrons for a single detected photon. They are so called Geiger mode Avalanche Photo Diodes (G-APD's). Our detectors are built in array comprised of a set of these components in order to be able to detect simultaneous photons. They are named Silicon Photo-Multiplier (SiPM) or Multi-Pixel Photon Counters (MPPC). Considering G-APD's of a diameter of 30 micrometers, a SiPM contains about one hundred diodes per square millimeters. However, these detectors are still too small -from one to three millimeters for commercial MPPC's- to be fully exploited in Imaging Atmospheric Cerenkov Telescope (IACT) experiments. Moreover, they have large and unavoidable dark noise [4], created by photon emissions inside the silicon lattice, which mainly depend on temperature and detector dimensions. For instance, the trigger frequency is typically of the order of $1 \mathrm{MHz} / \mathrm{mm}^{2}$, placed in complete darkness with a room temperature of $20^{\circ} \mathrm{C}$. For Cerenkov astronomy, dark noise is absolutely critical because of sky background which can reach several photons per pixel during a two nanoseconds acquisition. Thus a very poor signal-to-noise ratio is achieved. Because of this, major additional noise sources should be avoided. Obviously, thermal emission could be significantly reduced by cooling the detector. However, from an electronics point of view, large surfaces imply higher dielectric capacity, which means higher time response for avalanche trigger and a lower relaxation speed for 
G-APD's repolarization. For these reasons, SiPM surface enlargement is typically limited to 3 millimeters of diameter for our application.

There are then major issues connected to optical improvements while using this new technology based on SiPM when trying to achieve the same observing capabilities as with the PMT. This is precisely the object of the present communication where we present our simulation results for future Imaging Atmospheric Cerenkov Telescope in VHE gamma-ray astronomy.

\section{Optics placed upstream of the detection plane}

The weak photon flux encountered in VHE astronomy requires the use of several square meters of collection area for the primary mirror to focus the Cerenkov light onto camera pixels of square centimeters surface. Another requirement is that the field of view should be large and of the order of a few degrees. This implies that the primary reflector must have a focal over aperture diameter ( $f$ over $\mathrm{D}$ ) ratio close to unity to maintain a good image quality, a mechanical reliability, and to reduce delays due to the different light travel times $[6,7]$. For an optimal camera setup, the size of the spot onto the focal plane must be close to that of a pixel of the imager for a source placed at infinity. In this work, we concentrate primarily on a parabolic reflector. It is stigmatic along the optical axis and it has good ability to separate the directions of the incident light even with a short focal length but it suffers coma aberration. The optimized primary mirror in our simulation is concave, with a diameter of $4 \mathrm{~m}$ and a focal length of $4.8 \mathrm{~m}$. The incidence on the detection plane is then around $23^{\circ}$, which is a too large value when considering the constraints imposed by the detectors downstream from the system. It is indeed essential to be able to refocus the light independently on each pixel of the imager if one expects to focus a spot diameter of $15 \mathrm{~mm}$ on a $3 \mathrm{~mm} \mathrm{SiPM}$ and this can be done effectively only if the incident angle of the light on the detector is close to being perpendicular to the detector surface, with angles in the range of 8 to $9^{\circ}$.

It is therefore evident that the direct focus of the entire field of view on our millimetric detectors is impossible. For this reason we added a secondary convex parabolic mirror of $1 \mathrm{~m}$ diameter and $2.4 \mathrm{~m}$ focal length. In our simulation, we have chosen to place the detector location in the area engendered by the occultation of the primary mirror by the secondary, and we have evaluated that this optical configuration captures approximately $84 \%$-assuming a 6 $\%$ occultation factor and $90 \%$ reflectivity for the secondary mirror- of the flux received by a standard Cerenkov telescope of the same size with a single mirror. The optimal focus point has been computed -with the Zemax optical software [8] - to be at the primary reflector surface. Figure 1 shows the mirror configuration where ray traces have been displayed for different angles to illustrate the detection plane covering. A two degree filed represents a diameter of 40 centimeters on the camera. We now discuss the optics located in the detection plane. 
Fig. 1 Zemax simulation of a mirror configuration and ray traces for $0^{\circ}$ (blue),$+0.5^{\circ}$ (red) and $+1^{\circ}$ (green)

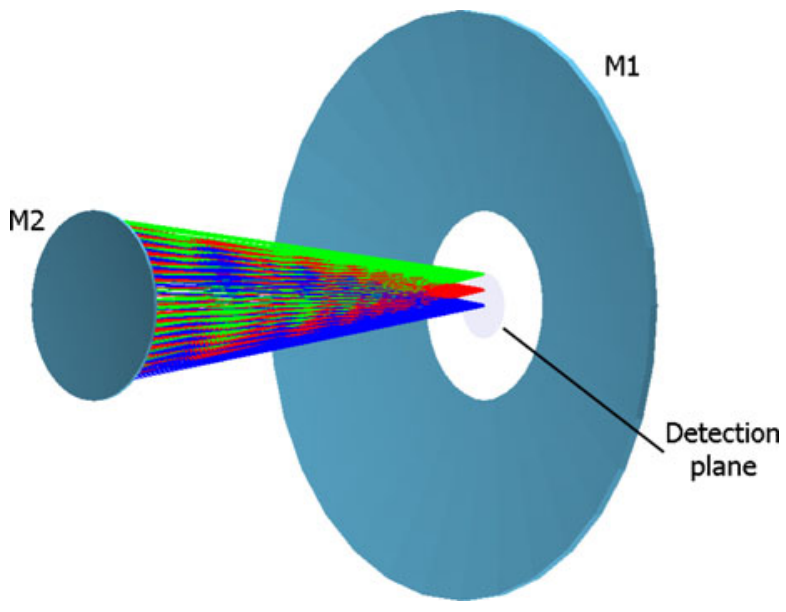

\section{Optics placed in the detection plane}

The detection plan consists of a $40 \mathrm{~cm}$ diameter camera of about 300 circular pixels. A single pixel corresponds to a single SiPM, the latter one containing a hundred G-APP's per square millimeter and a light concentrator as detailed below. Pixels are placed in a hexagonal grid to give an active area of $90.7 \%$ of the geometrical area. A square arrangement would give an active area of only $78.5 \%$, which explains why existing VHE projects, such as H.E.S.S. or VERITAS, implement this more efficient hexagonal packing $[9,10]$.

We first checked if the commonly used Winston's cone could be used to optimize the light collection in our setup. This cone is an optical transmission system, which combines the properties of a light concentrator and a cut-off angle system. The diameters of the entrance and exit faces are related to the maximum angle of acceptance which is typically of the order of 8 to $10^{\circ}$. Since the exit face area must match as close that of the detector as possible (which in our case is $3 \mathrm{~mm}$ ), the acceptance angle constraint translates to a typical diameter of $15 \mathrm{~mm}$ for the entrance face. Given this diameter, 300 pixels would have been required to cover the camera. However, as will be detailed below, we have considered the adjunction of microlenses to optimize the light collection on every G-APD of the SiPM. Winston's cone is of little interest in such a configuration as it deflects a sizable amount of light at large incidence (greater than $30^{\circ}$ ) even though in our case, the beam incidence from the secondary mirror does not exceed $9^{\circ}$. Instead of considering a Winston's cone, we have used an aspherical lens as a light concentrator from the pixel (typically $20 \mathrm{~mm}$ in length) to the SiPM. This is displayed in Fig. 2, where the light beam from the secondary mirror comes from the left. This Zemax simulation demonstrates the ability of large aperture lenses to refocus the mirror spot onto our detectors. Such lenses, including these diameter and focal length specifications, are commercially available. 


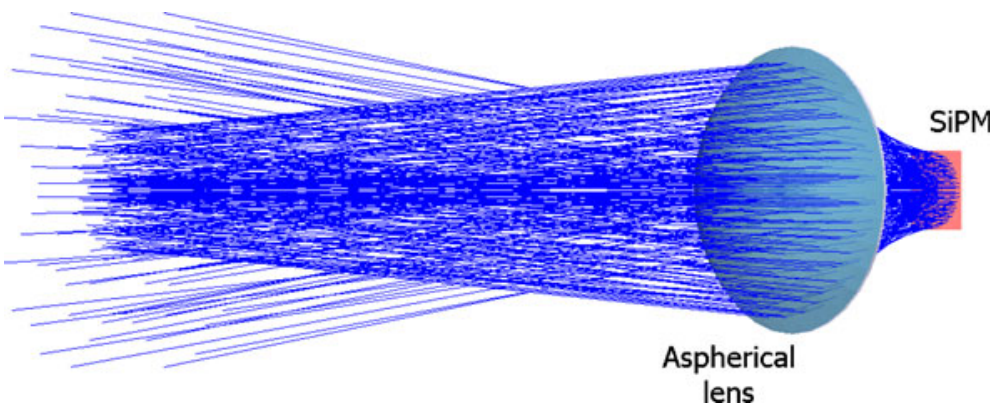

Fig. 2 Zemax simulation of an aspherical lens focusing a light beam onto a square SiPM

The global pixel layout can be seen in Fig. 3 where a single pixel is displayed. It is made of the lens described above, a fixation tube, a SiPM and its associated microlens array. Its parameters are calculated in such a way that the focus of the mirror image exactly matches the whole SiPM surface. The detection plane should not be directly exposed to the night sky because the stellar light could easily saturate the detectors. It is then necessary to limit the detector plane acceptance angle. Since the maximum size of the SiPM is constrained to be $3 \mathrm{~mm}$ because of the electronic noise and response time of the detector, and the incident angular distribution is given upstream, only the focal length is a free parameter. The lens diameter is provided by the spot size at the focal plane and therefore the number of pixel can be kept to a reasonably small number. The focal length $f$ of the aspherical lens cannot be reduced indefinitely because of aberration problems that may arise with an $f$ over $D$ ratio of less than unity. Given the various constraints of assembly (including mounting systems), the effective lens diameter has to be about $18 \mathrm{~mm}$ instead of the $20 \mathrm{~mm}$ assigned to each pixel thus, a loss of total area of $81 \%$.

As a last step in our study, we have subsequently considered the use and optimization of the microlenses by modeling once again the main parameters of the entire optical system. This included the glass, the different geometrical parameters and the pitch, which is the distance between two adjacent G-APD's. The goal was then to ensure that the light wave front arriving at each SiPM is mainly focused on sensitive areas of the APD's.

Fig. 3 Lens layout for refocusing on SiPM detector with microlenses: one pixel is displayed

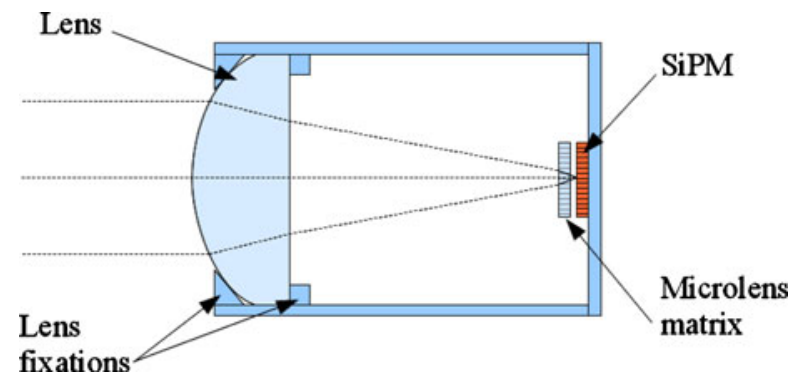




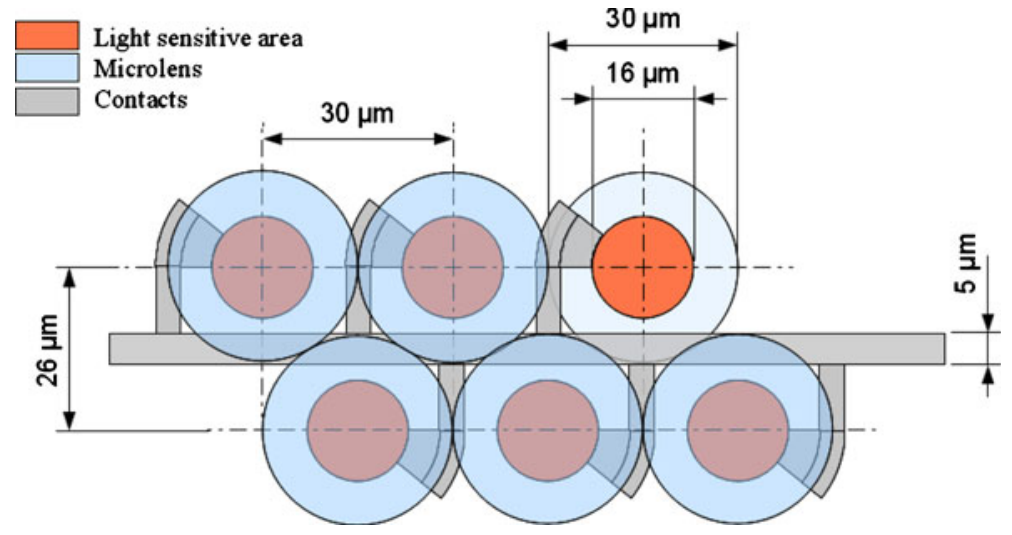

Fig. 4 SiPM layout including G-APD structure and microlenses (top view)

The silicon-based detectors have rather large quantum efficiency in contrast to conventional PMT's, but the drawback is that the photon detection efficiency is somewhat limited by the surface filling factor of typically $25 \%$ for a $30 \mu \mathrm{m}$ diode pitch. The building blocks of a SiPM cannot be placed in contact for trivial electrical reasons and therefore, a non negligible part of the surface is made up of 'dead' zones that are insensitive to an impinging photon. The provision of a microlens array aligned with the G-APD's significantly reduces the light losses. Commercially available microlens arrays comply with our particular arrangement as can be seen in Fig. 4 where G-APS's are directly etched onto the silicon wafer. As a matter of fact, the layout does not really matter until the individual diode structure is conserved and the electrical insulation is preserved.

Fig. 5 Optimization results for the microlens / G-APD coupling

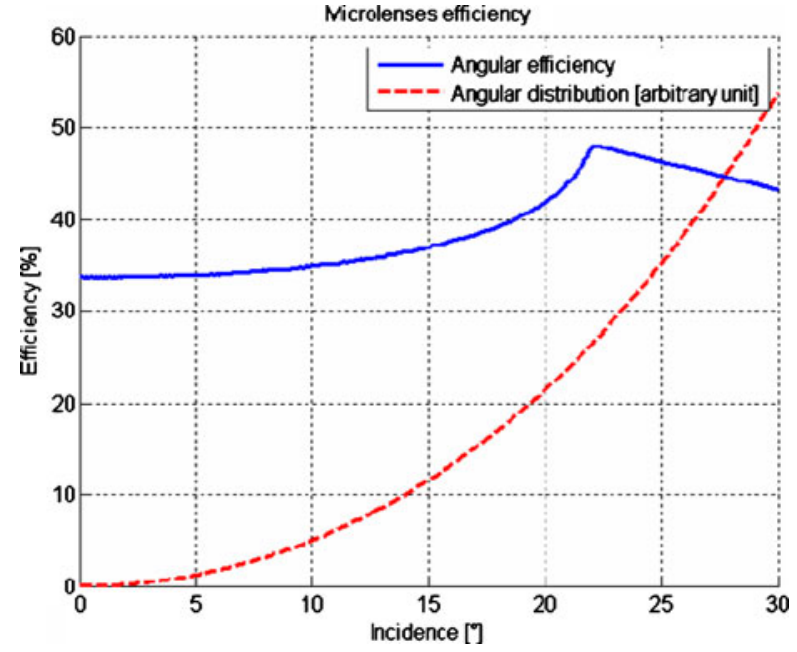


Table 1 Efficiency and incidence summary across the telescope and detectors

\begin{tabular}{|c|c|c|c|c|}
\hline \multicolumn{2}{|c|}{ Device / feature } & \multirow{2}{*}{$\begin{array}{l}\begin{array}{l}\text { Relative } \\
\text { eff. (\%) }\end{array} \\
90.0\end{array}$} & \multirow{2}{*}{$\begin{array}{l}\begin{array}{l}\text { Total } \\
\text { eff. (\%) }\end{array} \\
90.0\end{array}$} & \multirow{2}{*}{$\begin{array}{l}\begin{array}{l}\text { Max. } \\
\text { incidence }\left(^{\circ}\right)\end{array} \\
1\end{array}$} \\
\hline M1 & Reflectivity & & & \\
\hline \multirow[t]{2}{*}{ M2 } & Occultation & 93.8 & 84.4 & 23 \\
\hline & Reflectivity & 90.0 & 75.9 & \\
\hline Pixel & Hex. layout & 90.7 & 68.9 & 8 \\
\hline \multirow[t]{3}{*}{ Lens } & Transmission & 92.0 & - & \\
\hline & AR coating & 99.0 & 68.2 & \\
\hline & Mounting system & 81.0 & 51.3 & \\
\hline \multirow[t]{2}{*}{ Microlenses } & Transmission & 92.0 & - & 30 \\
\hline & AR coating & 99.0 & 50.8 & \\
\hline \multirow[t]{2}{*}{ SiPM } & Hex. layout & 90.7 & 46.1 & $>30$ \\
\hline & Active surface & 43.1 & 19.8 & \\
\hline
\end{tabular}

Unfortunately, adding these new optical modules will not ensure a perfect focus. Besides, the typical microlens focal length is generally one order of magnitude larger than needed. We have evaluated the effective lens surface at about $90.7 \%$ with a compact hexagonal configuration by using circular diodes. The 'geometrical' fill factor value is of $28.4 \%$ on the corresponding SiPM surface. However, the complete resolution of this light beam is not important, so that by placing microlenses very close to the SiPM surface at $6 \mu \mathrm{m}$, the light collection efficiency is substantially improved and it reaches $43.1 \%$. Figure 5 displays angular distribution onto the detector and light collection efficiency as a function of the incidence angle for a given microlens set. By integrating the product of these two quantities, one obtains a very relevant parameter to optimize light collection.

Despite its apparently poor global efficiency of $19.8 \%$, as displayed in Table 1, the presented system comprised with SiPM is still competitive
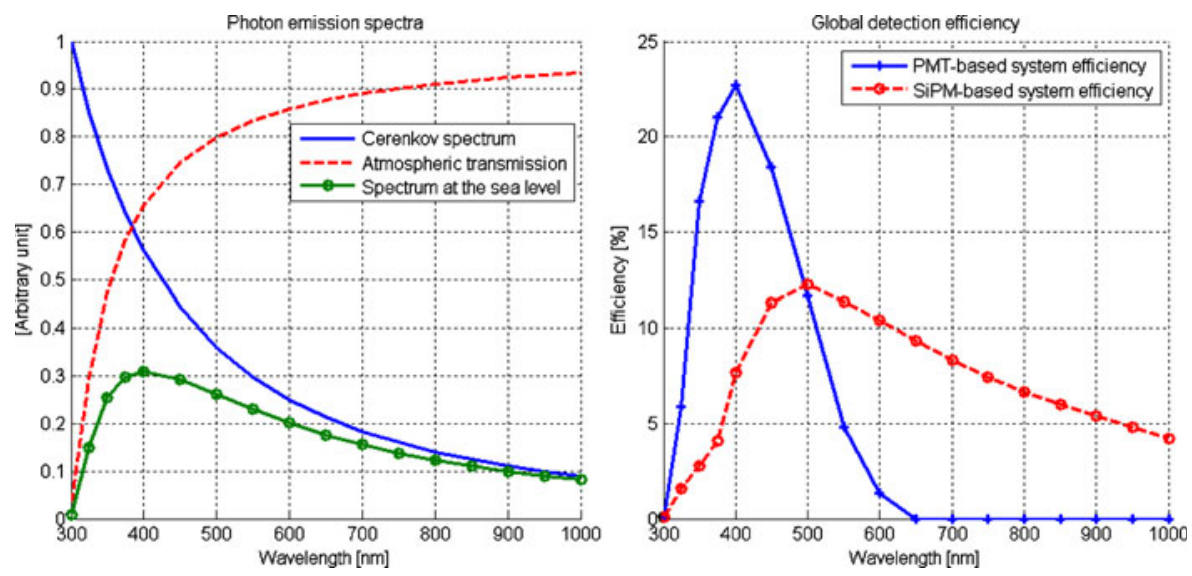

Fig. 6 Left Cerenkov emission spectrum. Right spectral comparison between PMT-based and SiPM-based systems for atmospheric Cerenkov detection 
compared to classical PMT-based systems, thanks to the really good quantum efficiency of the SiPM technology. Indeed, PMT's hardly reach a maximum of $30 \%$ for wavelengths in the range of $300-500 \mathrm{~nm}$ whereas silicon detectors could exceed $80 \%$ between 500 and $1,000 \mathrm{~nm}$ with a proper antireflection coating.

Figure 6 in its left side displays the Cerenkov emission spectrum, the atmospheric absorption and the resulting spectrum at the sea level. Taking into account this spectrum, PMT and SiPM quantum efficiencies and associated telescope optical systems properties, we have defined global detection efficiencies as a function of the wavelength as can be seen in the right side of Fig. 6. While integrating these curves over the 300 to $1,000 \mathrm{~nm}$ spectrum, we have obtained a mean detection efficiencies of about $7.5 \%$ for a SiPM-based system and only $5 \%$ for a PMT-based system. Therefore, one can make the point that the proposed system is in principle able to collect about $50 \%$ more light than a current IACT of the same size in addition to other advantages mentioned in the introduction. Consequently, SiPM would be a good candidate for VHE gamma-ray astronomy and unsurprisingly, other groups around the world have also started to develop camera based on these solid state detector $[11,12]$, with encouraging results.

\section{Conclusions}

In the framework of our $\mathrm{R} \& \mathrm{D}$ project that aims to develop SiPM detectors as alternatives to conventional PMT's used in VHE astronomy, we have carried out simulations to optimize the overall optical system. Our main findings are:

- a secondary mirror and light concentrators are required to focus a field of view of two degrees onto detectors with typical sizes of a few $\mathrm{mm}$ with incident angles of less than ten degrees,

- microlenses in front of G-APDs can provide an interesting solution to compensate for the relatively poor filling factors of available G-APDs.

These two major results could lead to better performances in large parks of small IACT's -typically four meters in diameter- like the Cerenkov Telescope Array program (CTA).

Acknowledgements The authors would like to thank Dr Natalie Webb for advice on the manuscript. This work was supported by Région Midi-Pyrénées and Observatoire Midi-Pyrénées (France).

\section{References}

1. Foord, R., Jones, R., Oliver, C.J., Pike, E.R.: The use of photomultiplier tubes for photon counting. Appl. Opt. 8(10), 1975-1989 (1969) 
2. Teich, M.C., Matsuo, K., Saleh, B.E.A.: Excess noise factors for conventional and superlattice avalanche photodiodes and photomultiplier tubes. IEEE J. Quantum Electron. QE-22(8), 1184-1193 (1986)

3. Pellion, D., Jradi, K., Le Padellec, A., Rennane, A., Moutier, F., Borrel, V., Esteve, D., Magenc, C., Bazer-Bachi, A.R.: Geiger Avalanche Photodiodes as tentative light detectors for VHE gamma ray astronomy. Exp. Astron. 27(3), 187-195 (2010)

4. Jradi, K., Pellion, D., Esteve, D., Boizard, J.-L., Le Padellec, A., Bazer-Bachi, A.R.: ComputerAided Design (CAD) Model for silicon avalanche Geiger mode systems design: application to high sensitivity imaging systems. Nucl. Instrum. Methods Phys. Res. Sect. A 626, 77-81 (2011)

5. Otono, H., Oide, H., Yamashita, S., Yoshioka, T.: On the basic mechanism of pixelized photon detectors. Nucl. Instrum. Methods Phys. Res. Sect. A 610, 397-399 (2009)

6. Bernlohr, K., Carrol, O., Cornils, R., Elfahem, S., Espigat, P., Gillessen, S., Heinzelmann, G., Hermann, G., Hofmann, W., Horns, D., Jung, I., Kankanyan, R., Katona, A., Khelifi, B., Krawczynski, H., Panter, M., Punch, M., Rayner, S., Rowell, G., Tluczykont, M., van Staa, R: The optical system of the HESS imaging atmospheric Cherenkov telescopes. Part I: layout and components of the system. Astropart. Phys. 20(2), 111-128 (2003)

7. Cornils, R., Gillessen, S., Jung, I., Hofmann, W., Beilicke, M., Bernlohr, K., Carrol, O., Elfahem, S., Heinzelmann, G., Hermann, G., Horns, D., Kankanyan, R., Katona, A., Krawczynski, H., Panter, M., Rayner, S., Rowell, G., Tluczykont, M., van Staa, R.: The optical system of the HESS imaging atmospheric Cherenkov telescopes. Part II: mirror alignment and point spread function. Astropart. Phys. 20(2), 129-143 (2003)

8. http://www.radiantzemax.com/en/design/

9. Hinton, J.A.: The status of the H.E.S.S. project. New Astron. Rev. 48(5-6), 331-337 (2004)

10. Holder, J., Atkins, R.W., Badran, H.M., Blaylock, G., Bradbury, S.M., Buckley, J.H., Byrum, K.L., Carter-Lewis, D.A., Celik, O., Chow, Y.C.K., Cogan, P., Cui, W., Daniel, M.K., Perez, I.D., Dowdall, C., Dowkontt, P., Duke, C., Falcone, A.D., Fegan, S.J., Finley, J.P., Fortin, P., Fortson, L.F., Gibbs, K., Gillanders, G., Glidewell, O.J., Grube, J., Gutierrez, K.J., Gyuk, G., Hall, J., Hanna, D., Hays, E., Horan, D., Hughes, S.B., Humensky, T.B., Imran, A., Jung, I., Kaaret, P., Kenny, G.E., Kieda, D., Kildea, J., Knapp, J., Krawczynski, H., Krennrich, F., Lang, M.J., LeBohec, S., Linton, E., Little, E.K., Maier, G., Manseri, H., Milovanovic, A., Moriarty, P., Mukherjee, R., Ogden, P.A., Ong, R.A., Petry, D., Perkins, J.S., Pizlo, F., Pohl, M., Quinn, J., Ragan, K., Reynolds, P.T., Roache, E.T., Rose, H.J., Schroedter, M., Sembroski, G.H., Sleege, G., Steele, D., Swordy, S.P., Syson, A., Toner, J.A., Valcarcel, L., Vassiliev, V.V., Wakely, S.P., Weekes, T.C., White, R.J., Williams, D.A., Wagner, R.: The first VERITAS telescope. Astropart. Phys. 25(6), 391-401 (2006)

11. Anderhub, H., Backes, M., Biland, A., Boller, A., Braun, I., Bretz, T., Commichau, S., Commichau, V., Dorner, D., Gendotti, A., Grimm, O., von Gunten, H., Hildebrand, D., Horisberger, U., Kohne, J.H., Krahenbuhl, T., Kranich, D., Lorenz, E., Lustermann, W., Mannheim, K., Neise, D., Pauss, F., Renker, D., Rhode, W., Rissi, M., Ribordy, M., Roser, U., Stark, L.S., Stucki, J.P., Tibolla, O., Viertel, G., Vogler, P., Weitzel, Q.: FACT-the first Cherenkoc telescope using a G-APD camera for TeV gamma-ray astronomy. Nucl. Instrum. Methods Phys. Res. Sect. A 639(1), 55-57 (2011)

12. Gamal, A., Bürler, P., Cargnelli, M., Hohler, R., Marton, J., Orth, H., Suzuki, K.: Application of Geiger-mode photosensors in Cherenkov detectors. Nucl. Instrum. Methods Phys. Res. Sect. A 639(1), 107-110 (2011) 